Acta Crystallographica Section E

Structure Reports

Online

ISSN 1600-5368

\section{2-Chloro-4-(3,3-dichloroallyloxy)-1- nitrobenzene}

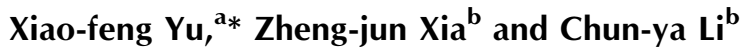

aschool of Pharmaceutics, Jiangsu University, Zhenjiang 212013, People's Republic of China, and ${ }^{\mathbf{b}}$ R\&D Center, Jiangsu Yabang Pharmaceutical Group, Liangchang Road East No. 6 Jingtan, Changzhou 213200, People's Republic of China

Correspondence e-mail: zhengfyu@126.com

Received 2 April 2012; accepted 25 April 2012

Key indicators: single-crystal X-ray study; $T=293 \mathrm{~K}$; mean $\sigma(\mathrm{C}-\mathrm{C})=0.007 \AA$; $R$ factor $=0.066 ; w R$ factor $=0.183 ;$ data-to-parameter ratio $=14.6$.

In the crystal structure of the title compound, $\mathrm{C}_{9} \mathrm{H}_{6} \mathrm{Cl}_{3} \mathrm{NO}_{3}$, molecules are connected by $\mathrm{C}-\mathrm{H} \cdots \mathrm{O}$ hydrogen bonds, forming chains along the $b$ axis. The dihedral angle between the benzene ring and the plane of the nitro group is $16.2(1)^{\circ}$ and that between the benzene ring and the plane of the dichloroallyl group is $10.2(1)^{\circ}$.

\section{Related literature}

For background to the applications of the title compound, see: Kolosov et al. (2002). For the synthesis, see: Walker et al. (2005).<smiles>O=[N+]([O-])c1ccc(OCC=C(Cl)Cl)cc1Cl</smiles>

\section{Experimental}

Crystal data

$\mathrm{C}_{9} \mathrm{H}_{6} \mathrm{Cl}_{3} \mathrm{NO}_{3} \quad M_{r}=282.50$
Monoclinic, $P 2_{1} / c$

$a=12.476(3) \AA$

$b=12.775(3) \AA$

$c=7.2230(14) \AA$

$\beta=92.32(3)^{\circ}$

$V=1150.3(4) \AA^{3}$

Data collection

Enraf-Nonius CAD-4 diffractometer

Absorption correction: $\psi$ scan (North et al., 1968)

$T_{\min }=0.799, T_{\max }=0.926$

2300 measured reflections

Refinement

$R\left[F^{2}>2 \sigma\left(F^{2}\right)\right]=0.066$

$w R\left(F^{2}\right)=0.183$

$S=1.00$

2118 reflections

$Z=4$

Mo $K \alpha$ radiation

$\mu=0.79 \mathrm{~mm}^{-1}$

$T=293 \mathrm{~K}$

$0.30 \times 0.20 \times 0.10 \mathrm{~mm}$

\author{
2118 independent reflections \\ 1414 reflections with $I>2 \sigma(I)$ \\ $R_{\text {int }}=0.023$ \\ 3 standard reflections every 200 \\ reflections \\ intensity decay: $1 \%$
}

Table 1

Hydrogen-bond geometry $\left(\AA{ }^{\circ}\right)$.

\begin{tabular}{lllll}
\hline$D-\mathrm{H} \cdots A$ & $D-\mathrm{H}$ & $\mathrm{H} \cdots A$ & $D \cdots A$ & $D-\mathrm{H} \cdots A$ \\
\hline $\mathrm{C} 5-\mathrm{H} 5 A \cdots \mathrm{O}^{\mathrm{i}}$ & 0.93 & 2.54 & $3.449(7)$ & 165 \\
\hline
\end{tabular}

Symmetry code: (i) $-x, y-\frac{1}{2},-z+\frac{1}{2}$.

Data collection: CAD-4 Software (Enraf-Nonius, 1985); cell refinement: $C A D-4$ Software; data reduction: XCAD4 (Harms \& Wocadlo, 1995); program(s) used to solve structure: SHELXS97 (Sheldrick, 2008); program(s) used to refine structure: SHELXS97 (Sheldrick, 2008); molecular graphics: SHELXTL (Sheldrick, 2008); software used to prepare material for publication: SHELXTL.

The authors thank the Center of Testing and Analysis, Nanjing University, for the data collection.

Supplementary data and figures for this paper are available from the IUCr electronic archives (Reference: VM2169).

\section{References}

Enraf-Nonius (1985). CAD-4 Software. Enraf-Nonius, Delft, The Netherlands.

Harms, K. \& Wocadlo, S. (1995). XCAD4. University of Marburg, Germany. Kolosov, S., Adamovich, V., Djurovich, P., Thompson, M. E. \& Adachi, C. (2002). J. Am. Chem. Soc. 124, 9945-9954.

North, A. C. T., Phillips, D. C. \& Mathews, F. S. (1968). Acta Cryst. A24, 351359.

Sheldrick, G. M. (2008). Acta Cryst. A64, 112-122.

Walker, E. R., Leung, S. Y. \& Barrett, A. G. M. (2005). Tetrahedron Lett. 46, 6537-6540. 


\section{supporting information}

Acta Cryst. (2012). E68, o1598 [doi:10.1107/S160053681201865X]

\section{2-Chloro-4-(3,3-dichloroallyloxy)-1-nitrobenzene}

\section{Xiao-feng Yu, Zheng-jun Xia and Chun-ya Li}

\section{S1. Comment}

The title compound is an important intermediate in the synthesis of phenanthrenes, which can be utilized to synthesize organic semiconductors and conjugated polymers (Walker et al., 2005). These materials are of wide current interest for applications in electronic and optoelectronic devices including light-emitting diodes (Kolosov et al., 2002). We report here the crystal structure of the title compound, (I), which is of interest to us in this field.

The molecular structure of (I) is shown in Fig. 1. There is an intermolecular contact $\mathrm{C}-\mathrm{H} \cdots \mathrm{O}$ in the title compound, forming molecular chains along the $b$ axis direction (Table 1, Fig. 2). These molecular chains are linked by weak $\pi-\pi$ interactions $\left(\mathrm{Cg} 1 \cdots \mathrm{Cg} 1^{\mathrm{i}}\right.$ distance $=3.724$ (3) $\AA, \mathrm{Cg} 1$ is the centroid of ring C1-C6, symmetry code: (i) $\left.x, 5 / 2-y,-1 / 2+z\right)$ to give a three-dimensional network, which seems to be very effective in the stabilization of the crystal structure.

The dihedral angles between the planes A (atoms $\mathrm{C} 1-\mathrm{C} 6$ ), $\mathrm{B}$ (atoms N/O2/O3), $\mathrm{C}$ (atoms $\mathrm{C} 7 / \mathrm{C} 8 / \mathrm{H} 8 \mathrm{~A} / \mathrm{C} 9 / \mathrm{Cl} / \mathrm{Cl} 3$ ) are: $\mathrm{A} / \mathrm{B}=16.2(1)^{\circ}, \mathrm{A} / \mathrm{C}=10.2(1)^{\circ}$.

\section{S2. Experimental}

The title compound, (I) was prepared by a method reported in literature (Walker et al., 2005). The crystals were obtained by dissolving (I) $(0.1 \mathrm{~g})$ in methanol $(30 \mathrm{ml})$ and evaporating the solvent slowly at room temperature for about $8 \mathrm{~d}$.

\section{S3. Refinement}

All $\mathrm{H}$ atoms were positioned geometrically and constrained to ride on their parent atoms, with $\mathrm{C}-\mathrm{H}=0.93 \AA$ for aromatic $\mathrm{H}$ and $0.96 \AA$ for alkyl $\mathrm{H}$, respectively. The $U_{\text {iso }}(\mathrm{H})=x U_{\mathrm{eq}}(\mathrm{C})$, where $x=1.2$ for aromatic $\mathrm{H}$ and $x=1.5$ for other H. 


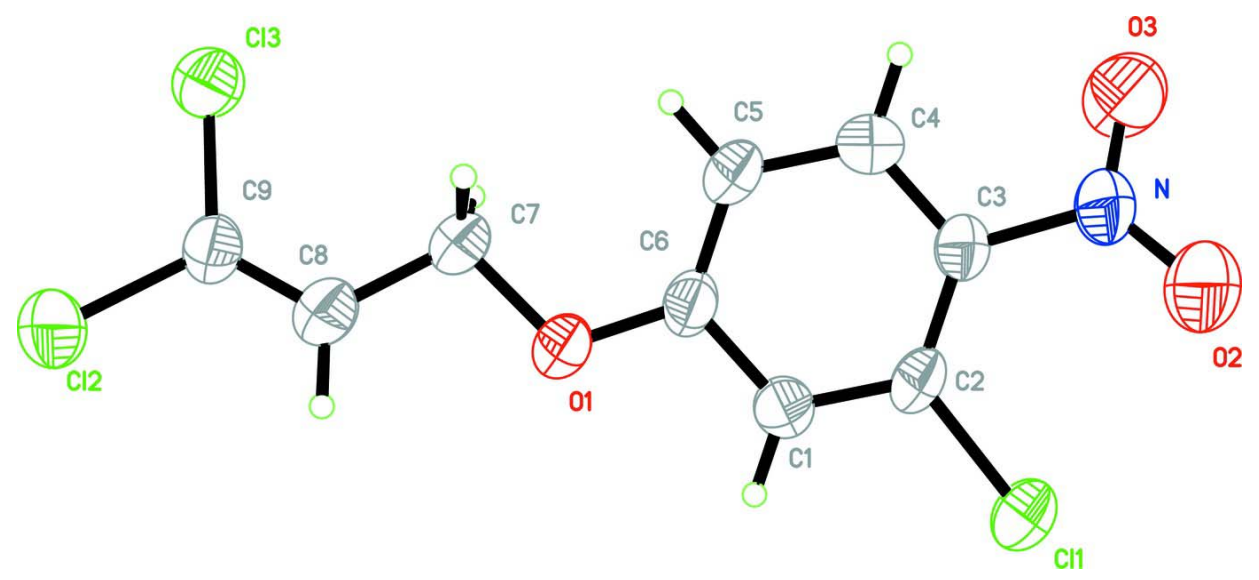

\section{Figure 1}

The molecular structure of (I), with the atom-numbering scheme. Displacement ellipsoids are drawn at the 50\% probability level.

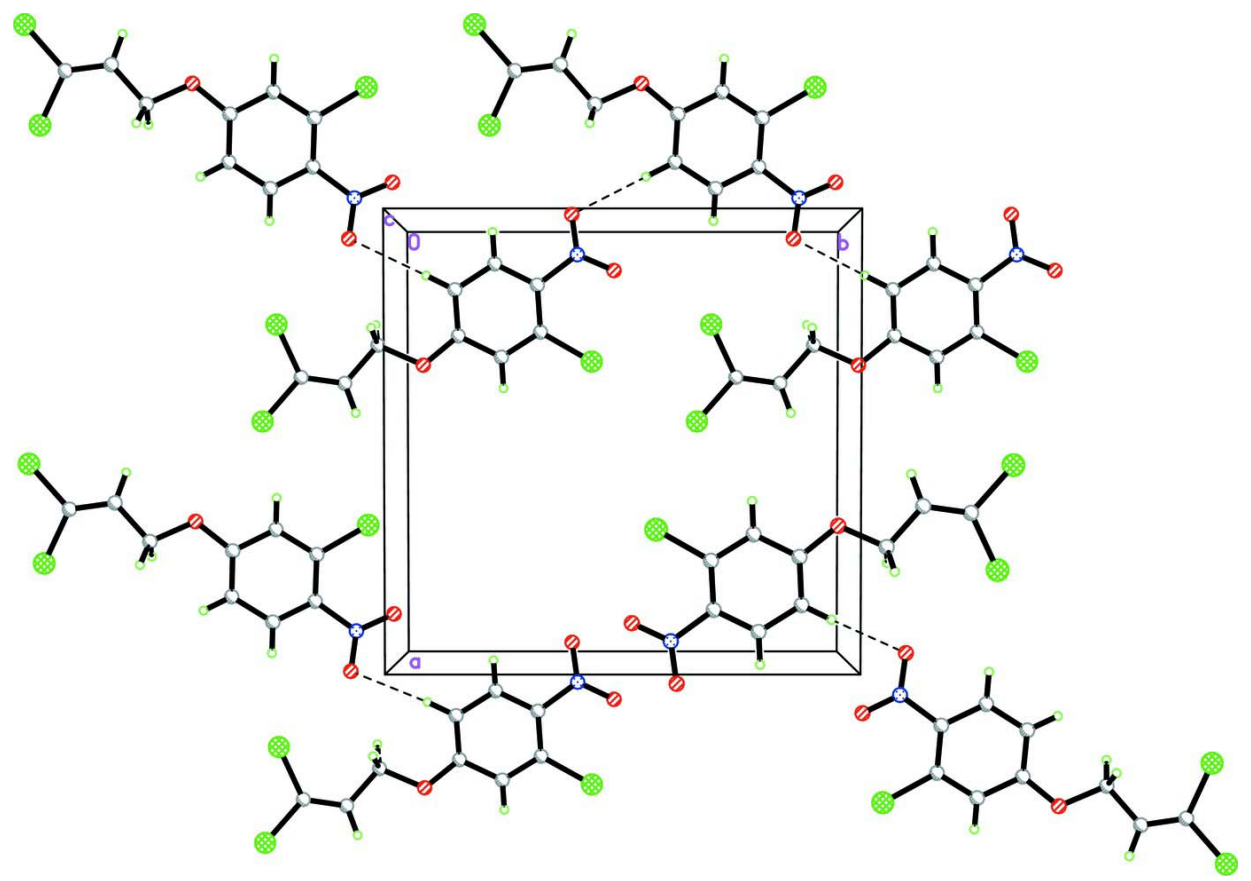

\section{Figure 2}

A packing diagram of (I) viewed along the $a$ axis ( $\mathrm{C}-\mathrm{H} \cdots \mathrm{O}$ hydrogen bonds are shown as broken lines).

\section{2-Chloro-4-(3,3-dichloroallyloxy)-1-nitrobenzene}

\section{Crystal data}

$\mathrm{C}_{9} \mathrm{H}_{6} \mathrm{Cl}_{3} \mathrm{NO}_{3}$

$M_{r}=282.50$

Monoclinic, $P 2_{1} / c$

Hall symbol: -P 2ybc

$a=12.476$ (3) $\AA$

$b=12.775(3) \AA$

$c=7.2230$ (14) $\AA$

$\beta=92.32(3)^{\circ}$

$$
\begin{aligned}
& V=1150.3(4) \AA^{3} \\
& Z=4 \\
& F(000)=568 \\
& D_{\mathrm{x}}=1.631 \mathrm{Mg} \mathrm{m}^{-3} \\
& \text { Mo } K \alpha \text { radiation, } \lambda=0.71073 \AA \\
& \text { Cell parameters from } 25 \text { reflections } \\
& \theta=10-13^{\circ} \\
& \mu=0.79 \mathrm{~mm}^{-1}
\end{aligned}
$$


$T=293 \mathrm{~K}$

Block, colourless

\section{Data collection}

\section{Enraf-Nonius CAD-4} diffractometer

Radiation source: fine-focus sealed tube Graphite monochromator $\omega / 2 \theta$ scans

Absorption correction: $\psi$ scan

(North et al., 1968)

$T_{\min }=0.799, T_{\max }=0.926$

2300 measured reflections

\section{Refinement}

Refinement on $F^{2}$

Least-squares matrix: full

$R\left[F^{2}>2 \sigma\left(F^{2}\right)\right]=0.066$

$w R\left(F^{2}\right)=0.183$

$S=1.00$

2118 reflections

145 parameters

0 restraints

Primary atom site location: structure-invariant direct methods
$0.30 \times 0.20 \times 0.10 \mathrm{~mm}$

2118 independent reflections

1414 reflections with $I>2 \sigma(I)$

$R_{\text {int }}=0.023$

$\theta_{\max }=25.4^{\circ}, \theta_{\min }=1.6^{\circ}$

$h=-15 \rightarrow 15$

$k=-15 \rightarrow 0$

$l=0 \rightarrow 8$

3 standard reflections every 200 reflections intensity decay: $1 \%$

Secondary atom site location: difference Fourier map

Hydrogen site location: inferred from neighbouring sites

$\mathrm{H}$-atom parameters constrained

$w=1 /\left[\sigma^{2}\left(F_{\mathrm{o}}{ }^{2}\right)+(0.1 P)^{2}+0.7 P\right]$

where $P=\left(F_{\mathrm{o}}{ }^{2}+2 F_{\mathrm{c}}{ }^{2}\right) / 3$

$(\Delta / \sigma)_{\max }<0.001$

$\Delta \rho_{\max }=0.52$ e $\AA^{-3}$

$\Delta \rho_{\min }=-0.42$ e $\AA^{-3}$

Special details

Geometry. All e.s.d.'s (except the e.s.d. in the dihedral angle between two 1.s. planes) are estimated using the full covariance matrix. The cell e.s.d.'s are taken into account individually in the estimation of e.s.d.'s in distances, angles and torsion angles; correlations between e.s.d.'s in cell parameters are only used when they are defined by crystal symmetry. An approximate (isotropic) treatment of cell e.s.d.'s is used for estimating e.s.d.'s involving 1.s. planes.

Refinement. Refinement of $F^{2}$ against ALL reflections. The weighted $R$-factor $w R$ and goodness of fit $S$ are based on $F^{2}$, conventional $R$-factors $R$ are based on $F$, with $F$ set to zero for negative $F^{2}$. The threshold expression of $F^{2}>\sigma\left(F^{2}\right)$ is used only for calculating $R$-factors(gt) etc. and is not relevant to the choice of reflections for refinement. $R$-factors based on $F^{2}$ are statistically about twice as large as those based on $F$, and $R$ - factors based on ALL data will be even larger.

Fractional atomic coordinates and isotropic or equivalent isotropic displacement parameters $\left(\AA^{2}\right)$

\begin{tabular}{lllll}
\hline & $x$ & $y$ & $z$ & $U_{\text {iso }} * / U_{\text {eq }}$ \\
\hline $\mathrm{N}$ & $0.0625(4)$ & $1.3968(3)$ & $0.1971(7)$ & $0.0603(12)$ \\
$\mathrm{Cl1}$ & $0.31002(11)$ & $1.42882(9)$ & $0.1250(2)$ & $0.0643(4)$ \\
$\mathrm{C} 1$ & $0.2996(4)$ & $1.2216(4)$ & $0.1245(7)$ & $0.0468(11)$ \\
$\mathrm{H} 1 \mathrm{~A}$ & 0.3724 & 1.2256 & 0.1021 & $0.056^{*}$ \\
$\mathrm{O} 1$ & $0.3184(3)$ & $1.0417(2)$ & $0.1211(5)$ & $0.0560(9)$ \\
$\mathrm{Cl} 2$ & $0.45065(11)$ & $0.67097(10)$ & $0.1106(2)$ & $0.0685(5)$ \\
$\mathrm{C} 2$ & $0.2415(4)$ & $1.3117(3)$ & $0.1436(7)$ & $0.0452(11)$ \\
$\mathrm{O} 2$ & $0.1009(4)$ & $1.4802(3)$ & $0.2323(8)$ & $0.1053(18)$ \\
$\mathrm{C} 13$ & $0.22239(11)$ & $0.70364(10)$ & $0.1046(2)$ & $0.0679(5)$ \\
$\mathrm{C} 3$ & $0.1325(3)$ & $1.3051(3)$ & $0.1756(7)$ & $0.0441(11)$ \\
$\mathrm{O} 3$ & $-0.0309(3)$ & $1.3841(4)$ & $0.1923(12)$ & $0.157(3)$ \\
$\mathrm{C} 4$ & $0.0837(4)$ & $1.2084(4)$ & $0.1880(7)$ & $0.0505(12)$ \\
$\mathrm{H} 4 \mathrm{~A}$ & 0.0105 & 1.2044 & 0.2073 & $0.061 *$
\end{tabular}




$\begin{array}{lllll}\text { C5 } & 0.1433(4) & 1.1170(4) & 0.1718(7) & 0.0496(12) \\ \text { H5A } & 0.1106 & 1.0520 & 0.1837 & 0.060^{*} \\ \text { C6 } & 0.2501(4) & 1.1231(3) & 0.1384(6) & 0.0432(11) \\ \text { C7 } & 0.2718(4) & 0.9380(3) & 0.1296(8) & 0.0585(14) \\ \text { H7A } & 0.2213 & 0.9272 & 0.0256 & 0.070^{*} \\ \text { H7B } & 0.2340 & 0.9297 & 0.2434 & 0.070^{*} \\ \text { C8 } & 0.3611(4) & 0.8612(4) & 0.1230(7) & 0.0542(13) \\ \text { H8A } & 0.4310 & 0.8865 & 0.1258 & 0.065^{*} \\ \text { C9 } & 0.3467(4) & 0.7601(4) & 0.1137(7) & 0.0493(12)\end{array}$

Atomic displacement parameters $\left(\AA^{2}\right)$

\begin{tabular}{lllllll}
\hline & $U^{11}$ & $U^{22}$ & $U^{33}$ & $U^{12}$ & $U^{13}$ & $U^{23}$ \\
\hline $\mathrm{N}$ & $0.055(3)$ & $0.040(2)$ & $0.087(3)$ & $0.0090(19)$ & $0.005(2)$ & $-0.007(2)$ \\
$\mathrm{C} 11$ & $0.0614(8)$ & $0.0331(6)$ & $0.0991(11)$ & $-0.0085(5)$ & $0.0112(7)$ & $-0.0018(6)$ \\
$\mathrm{C} 1$ & $0.042(2)$ & $0.037(2)$ & $0.062(3)$ & $0.0005(19)$ & $0.013(2)$ & $0.005(2)$ \\
$\mathrm{O} 1$ & $0.0505(18)$ & $0.0324(16)$ & $0.086(2)$ & $-0.0030(14)$ & $0.0182(17)$ & $0.0000(17)$ \\
$\mathrm{C} 2$ & $0.0583(8)$ & $0.0419(7)$ & $0.1055(12)$ & $0.0096(6)$ & $0.0065(7)$ & $-0.0031(7)$ \\
$\mathrm{C} 2$ & $0.052(3)$ & $0.026(2)$ & $0.058(3)$ & $-0.0048(19)$ & $0.005(2)$ & $-0.001(2)$ \\
$\mathrm{O} 2$ & $0.081(3)$ & $0.042(2)$ & $0.194(6)$ & $0.011(2)$ & $0.022(3)$ & $-0.013(3)$ \\
$\mathrm{C} 3$ & $0.0563(8)$ & $0.0419(7)$ & $0.1063(12)$ & $-0.0047(6)$ & $0.0121(7)$ & $-0.0094(7)$ \\
$\mathrm{C} 3$ & $0.044(2)$ & $0.031(2)$ & $0.058(3)$ & $0.0050(19)$ & $0.002(2)$ & $0.000(2)$ \\
$\mathrm{O} 3$ & $0.037(2)$ & $0.069(3)$ & $0.366(10)$ & $0.009(2)$ & $0.019(4)$ & $-0.050(5)$ \\
$\mathrm{C} 4$ & $0.041(2)$ & $0.046(3)$ & $0.065(3)$ & $-0.004(2)$ & $0.010(2)$ & $-0.001(2)$ \\
$\mathrm{C} 5$ & $0.054(3)$ & $0.030(2)$ & $0.066(3)$ & $-0.005(2)$ & $0.011(2)$ & $0.003(2)$ \\
$\mathrm{C} 6$ & $0.051(3)$ & $0.029(2)$ & $0.050(3)$ & $0.0001(19)$ & $0.010(2)$ & $0.001(2)$ \\
$\mathrm{C} 7$ & $0.048(3)$ & $0.032(2)$ & $0.096(4)$ & $-0.006(2)$ & $0.010(3)$ & $-0.002(3)$ \\
$\mathrm{C} 8$ & $0.052(3)$ & $0.037(3)$ & $0.074(3)$ & $-0.005(2)$ & $0.006(2)$ & $-0.002(2)$ \\
$\mathrm{C} 9$ & $0.052(3)$ & $0.037(3)$ & $0.059(3)$ & $0.004(2)$ & $0.008(2)$ & $0.006(2)$ \\
& & & & & & \\
\hline
\end{tabular}

Geometric parameters $\left(A,{ }^{\circ}\right)$

\begin{tabular}{llll}
\hline $\mathrm{N}-\mathrm{O} 3$ & $1.176(6)$ & $\mathrm{Cl}-\mathrm{C} 9$ & $1.709(5)$ \\
$\mathrm{N}-\mathrm{O} 2$ & $1.192(6)$ & $\mathrm{C} 3-\mathrm{C} 4$ & $1.382(6)$ \\
$\mathrm{N}-\mathrm{C} 3$ & $1.472(6)$ & $\mathrm{C} 4-\mathrm{C} 5$ & $1.392(6)$ \\
$\mathrm{C} 11-\mathrm{C} 2$ & $1.731(4)$ & $\mathrm{C} 4-\mathrm{H} 4 \mathrm{~A}$ & 0.9300 \\
$\mathrm{C} 1-\mathrm{C} 2$ & $1.370(6)$ & $\mathrm{C} 5-\mathrm{C} 6$ & $1.367(6)$ \\
$\mathrm{C} 1-\mathrm{C} 6$ & $1.406(6)$ & $\mathrm{C} 5-\mathrm{H} 5 \mathrm{~A}$ & 0.9300 \\
$\mathrm{C} 1-\mathrm{H} 1 \mathrm{~A}$ & 0.9300 & $\mathrm{C} 7-\mathrm{C} 8$ & $1.487(6)$ \\
$\mathrm{O} 1-\mathrm{C} 6$ & $1.353(5)$ & $\mathrm{C} 7-\mathrm{H} 7 \mathrm{~A}$ & 0.9700 \\
$\mathrm{O} 1-\mathrm{C} 7$ & $1.449(5)$ & $\mathrm{C} 7-\mathrm{H} 7 \mathrm{~B}$ & 0.9700 \\
$\mathrm{C} 12-\mathrm{C} 9$ & $1.727(5)$ & $\mathrm{C} 8-\mathrm{C} 9$ & $1.304(7)$ \\
$\mathrm{C} 2-\mathrm{C} 3$ & $1.392(6)$ & $\mathrm{C} 8-\mathrm{H} 8 \mathrm{~A}$ & 0.9300 \\
& & & 120.2 \\
$\mathrm{O} 3-\mathrm{N}-\mathrm{O} 2$ & $121.2(5)$ & $\mathrm{C} 6-\mathrm{C} 5-\mathrm{H} 5 \mathrm{~A}$ & 120.2 \\
$\mathrm{O} 3-\mathrm{N}-\mathrm{C} 3$ & $118.6(4)$ & $\mathrm{C} 4-\mathrm{C} 5-\mathrm{H} 5 \mathrm{~A}$ & $126.4(4)$ \\
$\mathrm{O} 2-\mathrm{N}-\mathrm{C} 3$ & $119.9(4)$ & $\mathrm{O} 1-\mathrm{C} 6-\mathrm{C} 5$ & $113.6(4)$ \\
$\mathrm{C} 2-\mathrm{C} 1-\mathrm{C} 6$ & $120.6(4)$ & $\mathrm{O} 1-\mathrm{C} 6-\mathrm{C} 1$ &
\end{tabular}




$\begin{array}{llll}\mathrm{C} 2-\mathrm{C} 1-\mathrm{H} 1 \mathrm{~A} & 119.7 & \mathrm{C} 5-\mathrm{C} 6-\mathrm{C} 1 & 119.9(4) \\ \mathrm{C} 6-\mathrm{C} 1-\mathrm{H} 1 \mathrm{~A} & 119.7 & \mathrm{O} 1-\mathrm{C} 7-\mathrm{C} 8 & 107.5(4) \\ \mathrm{C} 6-\mathrm{O} 1-\mathrm{C} 7 & 116.4(4) & \mathrm{O} 1-\mathrm{C} 7-\mathrm{H} 7 \mathrm{~A} & 110.2 \\ \mathrm{C} 1-\mathrm{C} 2-\mathrm{C} 3 & 119.4(4) & \mathrm{C} 8-\mathrm{C} 7-\mathrm{H} 7 \mathrm{~A} & 110.2 \\ \mathrm{C} 1-\mathrm{C} 2-\mathrm{C} 11 & 117.0(4) & \mathrm{O} 1-\mathrm{C} 7-\mathrm{H} 7 \mathrm{~B} & 110.2 \\ \mathrm{C} 3-\mathrm{C} 2-\mathrm{C} 11 & 123.6(3) & \mathrm{C} 8-\mathrm{C} 7-\mathrm{H} 7 \mathrm{~B} & 108.5 \\ \mathrm{C} 4-\mathrm{C} 3-\mathrm{C} 2 & 120.1(4) & \mathrm{H} 7 \mathrm{~A}-\mathrm{C} 7-\mathrm{H} 7 \mathrm{~B} & 123.6(5) \\ \mathrm{C} 4-\mathrm{C} 3-\mathrm{N} & 116.1(4) & \mathrm{C} 9-\mathrm{C} 8-\mathrm{C} 7 & 118.2 \\ \mathrm{C} 2-\mathrm{C} 3-\mathrm{N} & 123.9(4) & \mathrm{C} 9-\mathrm{C} 8-\mathrm{H} 8 \mathrm{~A} & 118.2 \\ \mathrm{C} 3-\mathrm{C} 4-\mathrm{C} 5 & 120.4(4) & \mathrm{C} 7-\mathrm{C} 8-\mathrm{H} 8 \mathrm{~A} & 122.9(4) \\ \mathrm{C} 3-\mathrm{C} 4-\mathrm{H} 4 \mathrm{~A} & 119.8 & \mathrm{C} 8-\mathrm{C} 9-\mathrm{C} 3 & 123.4(4) \\ \mathrm{C} 5-\mathrm{C} 4-\mathrm{H} 4 \mathrm{~A} & 119.8 & \mathrm{C} 8-\mathrm{C} 9-\mathrm{C} 2 & 113.7(3) \\ \mathrm{C} 6-\mathrm{C} 5-\mathrm{C} 4 & 119.7(4) & \mathrm{C} 3-\mathrm{C} 9-\mathrm{C} 2 & -1.8(7) \\ & & & 3.3(7) \\ \mathrm{C} 6-\mathrm{C} 1-\mathrm{C} 2-\mathrm{C} 3 & \mathrm{C} 3-\mathrm{C} 4-\mathrm{C} 5-\mathrm{C} 6 & -178.5(4) \\ \mathrm{C} 6-\mathrm{C} 1-\mathrm{C} 2-\mathrm{C} 11 & 179.8(4) & \mathrm{C} 7-\mathrm{O} 1-\mathrm{C} 6-\mathrm{C} 5 & 179.4(5) \\ \mathrm{C} 1-\mathrm{C} 2-\mathrm{C} 3-\mathrm{C} 4 & 0.0(7) & \mathrm{C} 7-\mathrm{O} 1-\mathrm{C} 6-\mathrm{C} 1 & 1.3(7) \\ \mathrm{C} 11-\mathrm{C} 2-\mathrm{C} 3-\mathrm{C} 4 & 179.8(4) & \mathrm{C} 4-\mathrm{C} 5-\mathrm{C} 6-\mathrm{O} 1 & -178.4(4) \\ \mathrm{C} 1-\mathrm{C} 2-\mathrm{C} 3-\mathrm{N} & -179.6(5) & \mathrm{C} 4-\mathrm{C} 5-\mathrm{C} 6-\mathrm{C} 1 & -0.2(7) \\ \mathrm{C} 11-\mathrm{C} 2-\mathrm{C} 3-\mathrm{N} & 0.1(7) & \mathrm{C} 2-\mathrm{C} 1-\mathrm{C} 6-\mathrm{O} 1 & -175.5(4) \\ \mathrm{O} 3-\mathrm{N}-\mathrm{C} 3-\mathrm{C} 4 & -12.3(8) & \mathrm{C} 2-\mathrm{C} 1-\mathrm{C} 6-\mathrm{C} 5 & -174.5(5) \\ \mathrm{O} 2-\mathrm{N}-\mathrm{C} 3-\mathrm{C} 4 & 162.1(5) & \mathrm{C} 6-\mathrm{O} 1-\mathrm{C} 7-\mathrm{C} 8 & 0.9(8) \\ \mathrm{O} 3-\mathrm{N}-\mathrm{C} 3-\mathrm{C} 2 & 167.3(6) & \mathrm{O} 1-\mathrm{C} 7-\mathrm{C} 8-\mathrm{C} 9 & -178.7(4) \\ \mathrm{O} 2-\mathrm{N}-\mathrm{C} 3-\mathrm{C} 2 & -18.2(8) & \mathrm{C} 7-\mathrm{C} 8-\mathrm{C} 9-\mathrm{C} 13 & \\ \mathrm{C} 2-\mathrm{C} 3-\mathrm{C} 4-\mathrm{C} 5 & 1.1(7) & \mathrm{C} 7-\mathrm{C} 8-\mathrm{C} 9-\mathrm{C} 2 & \\ \mathrm{~N}-\mathrm{C} 3-\mathrm{C} 4-\mathrm{C} 5 & -179.2(5) & & \end{array}$

Hydrogen-bond geometry $\left(\AA,{ }^{\circ}\right)$

\begin{tabular}{lllll}
\hline$D-\mathrm{H} \cdots A$ & $D-\mathrm{H}$ & $\mathrm{H} \cdots A$ & $D \cdots A$ & $D-\mathrm{H} \cdots A$ \\
\hline $\mathrm{C} 5-\mathrm{H} 5 A \cdots \mathrm{O} 3^{\mathrm{i}}$ & 0.93 & 2.54 & $3.449(7)$ & 165 \\
\hline
\end{tabular}

Symmetry code: (i) $-x, y-1 / 2,-z+1 / 2$. 